\title{
The emerging role of circular RNAs in common solid malignant tumors in children
}

\author{
Jiabin Yu ${ }^{1,2}$ (D) Li Yang ${ }^{1,2}$ and Hongting Lu²*
}

\begin{abstract}
Malignant tumors are one of the fatal diseases that threaten children's physical and mental health and affect their development. Research has shown that the occurrence and development of malignant tumors are associated with the abnormal expression and regulation of genes. Circular RNAs (circRNAs) are noncoding RNAs that have a closed circular structure, with a relatively stable expression, and do not undergo exonuclease-mediated degradation readily. Recent studies have shown that circRNA plays an important role in the occurrence, metastasis, and invasion of solid malignant tumors (SMTs) in children. Thus, circRNA is being considered as a breakthrough in the treatment of SMTs in children. In this review, we describe the functions and mechanisms of circRNAs involved in SMTs in children oncogenesis, and summarize the roles of circRNAs in regulating cell proliferation, cell apoptotic death, the cell cycle, cell migrative and invasive ability, epithelial-mesenchymal transition (EMT), cancer stem cells and drug resistance in SMTs in children. In addition, we also discuss the role of circRNAs in the early diagnosis, pathological grading, targeted therapy, and prognosis evaluation of common SMTs in children. CircRNAs are likely to provide a novel direction in therapy in SMTs of children.
\end{abstract}

Keywords: circRNA, Children, Malignant tumors, Solid tumors, Differential expression

\section{Introduction}

After accidental deaths, malignant tumors constitute one of the critical reasons for childhood deaths and are a serious threat to children's health [1-2]. The solid malignant tumors (SMTs) in children mainly occur in the central, sympathetic nervous system, and mesenchymal tissues. Common ones include osteosarcoma, glioma, neuroblastoma, hepatoblastoma, infantile hemangioma, rhabdomyosarcoma, retinoblastoma, Wilms tumor, medulloblastoma and ependymoma, etc. The treatment methods include surgical resection, chemotherapy, and radiation therapy, but the treatment results are generally not favorable. Therefore, it is particularly important to explore new biomarkers and molecular mechanisms to treat SMTs in children. Several studies have shown

\footnotetext{
*Correspondence: luhongting@126.com

2 Surgical Center of Women and Children's Hospital, Qingdao University, No. 6, Tongfu Rd, Shibei District, Qingdao 266011, Shandong, China

Full list of author information is available at the end of the article
}

that tumor development, cell invasion, proliferation, and apoptosis are closely related to the expression and regulation of noncoding RNAs. CircRNAs constitute a type of stable noncoding RNAs typically found in the cytoplasm of eukaryotic cells; however, a small part of intron-derived circRNA can be found in the nucleus. The traditional linear RNAs contain a $5^{\prime}$ end cap and a $3^{\prime}$ end poly(A) tail, but circRNAs have a closed-loop structure without $5^{\prime}$ and $3^{\prime}$ ends, have stable expression, and do not undergo endonuclease-mediated degradation readily [3]. Studies have shown that many circRNAs are differentially expressed in common SMTs in children and are involved in the occurrence and development of tumors. In this review, we will summarize the functions and mechanisms of circRNAs in SMTs in children oncogenesis and malignant progression.

\section{Overview of circRNA}

CircRNA was first discovered by Sanger et al. [4] in RNA viruses in 1976. With the rapid development of

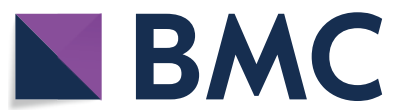

(c) The Author(s) 2021. This article is licensed under a Creative Commons Attribution 4.0 International License, which permits use, sharing, adaptation, distribution and reproduction in any medium or format, as long as you give appropriate credit to the original author(s) and the source, provide a link to the Creative Commons licence, and indicate if changes were made. The images or other third party material in this article are included in the article's Creative Commons licence, unless indicated otherwise in a credit line to the material. If material is not included in the article's Creative Commons licence and your intended use is not permitted by statutory regulation or exceeds the permitted use, you will need to obtain permission directly from the copyright holder. To view a copy of this licence, visit http://creativeco mmons.org/licenses/by/4.0/. The Creative Commons Public Domain Dedication waiver (http://creativecommons.org/publicdomain/ zero/1.0/) applies to the data made available in this article, unless otherwise stated in a credit line to the data. 
high-throughput sequencing techniques, in 2012, Salzman et al. [5] were the first ones to detect more than 80 circRNAs in human leukocytes using RNA sequencing methods. Subsequently, Jeck et al. [6] discovered more than 25,000 circRNAs in human fibroblasts. Such a huge amount of data indicated that circRNAs were not accidental products during RNA splicing but were widely present, stable, and highly conserved in human cells. Most circRNAs are formed by reverse splicing of exons; however, a small part is formed by introns and intergenic regions. Based on their source, they are divided into exonic circRNAs (derived from exons) [7], intronic circRNAs (derived from introns) [8], exon-intron circRNAs (derived from exons and introns) [9], etc. Additionally, there exists a special type of circRNA, called tricRNA, which is formed by splicing from the precursor tRNA [10]. Studies have found that its expression is agedependent and tissue-specific.

\section{Biological functions of circRNA MiRNA sponge effect}

CircRNAs absorb miRNA, like a sponge, via base complementation, and is referred to as the "miRNA sponge effect." In 2013, two research teams reported that CDR1derived circRNA (ciRS-7) could bind to and adsorb miR-7, reduce its activity, and indirectly upregulate the expression of miR-7-related target genes [11]. Zheng et al. found that circHIPK 3 could directly bind to miR124 and inhibit its activity [12]. In recent years, several studies have confirmed that the miRNA sponge effect of circRNA can be observed in various tissues and cell lines. Due to the stable structure of circRNAs, they exhibit better adsorption of miRNA than linear RNAs.

One of the essential functions of circRNAs includes transcriptional regulation. Li et al. [9] used cross-linked immunoprecipitation to study the effect of circRNA regulation on the transcription mechanism and identified the interaction between 111 circRNAs and RNA polymerase II in HeLa cells. CircEIF3J and CircPAIP2 knockout in HeLa and HEK293 cells, respectively, reduced the mRNA expression of the parent gene EIF3J and PAIP2. Also, the FMN gene is vital for limb development in mice. The exon circRNA is generated by reverse splicing during FMN gene transcription. Mice lacking this scissor acceptor are unable to detect exon circRNA expression. Although they have normal limb development, they will eventually develop the incomplete penetrance phenotype of renal hypoplasia [13]. CircRNAs reduce protein expression by blocking the translation start sites.

\section{Interact with RNA binding proteins (RBP)}

A study found that circRNAs can directly combine with RNAbinding proteins (RBP) to form an RNA-protein complex, which can regulate RBP or directly act on target genes via partial base pairing. They also interact with proteins indirectly via RNA-mediated and affect protein functions [14]. For example, circRNAs form a stable bond with AGO protein and RNA polymerase II and enhance gene transcription by increasing its transcription function. Exon circRNAs also serve as a "scaffold" for RNAbinding proteins by binding to multiple proteins to strengthen the stability of their interactions. Studies have also suggested that circRNAs can be used as a targeting element while binding to RBP and complementary RNA or DNA sequences [7]. The splicing factor MBL is a type of RBP. In humans and Drosophila, it can combine with the second exon of its parent gene to promote its cyclization to form circMbl, which can combine with MBL, reduce the effective concentration of MBL, ultimately reducing the synthesis of circMbl [15].

\section{Participate in protein translation}

Some circRNAs can encode proteins. The hepatitis delta factor, present in mammalian cells, is a protein encoded by circRNA [16]. Studies have shown that circRNAs in U20S, a human osteosarcoma cell line, exhibit low-efficiency protein translation [17]. Legnini reported that circ-ZNF609 could bind to polyribosomes and participate in the transcription and translation of proteins through $5^{\prime}$ cap-independent and splicing-independent forms [18].

\section{CircRNA and common SMTs in children}

In the following sections, we summarize the current research on the specific role and mechanism of circular RNA in the progression of SMTs in children (Fig, 1, Table 1).

\section{Osteosarcoma}

Osteosarcoma (OS) is a type of malignant bone tumor commonly found in adolescents or children. It constitutes approximately $5 \%$ of pediatric tumors. Yang et al. [19] tested the cancerous tissues, paracancerous tissues, and cell lines (U2OS, MG63) of 30 OS patients using qRT-PCR and found a significantly lower expression of circ_0001105 and YTHDF2 in OS tissues and cell lines compared with paracancerous tissues, while miR-766 levels were substantially elevated than the adjacent tissue. Also, the expression of circ_0001105 in patients with advanced cancer, metastatic tumors, and recurrent tumors was considerably higher, which indicated that downregulated expression of circ_0001105 was related to the prognosis and survival of patients. Regarding functional tests, ectopic overexpression of circ_0001105 or YTHDF2 significantly inhibited the proliferation, migration, and invasion of OS cells via the regulation of 


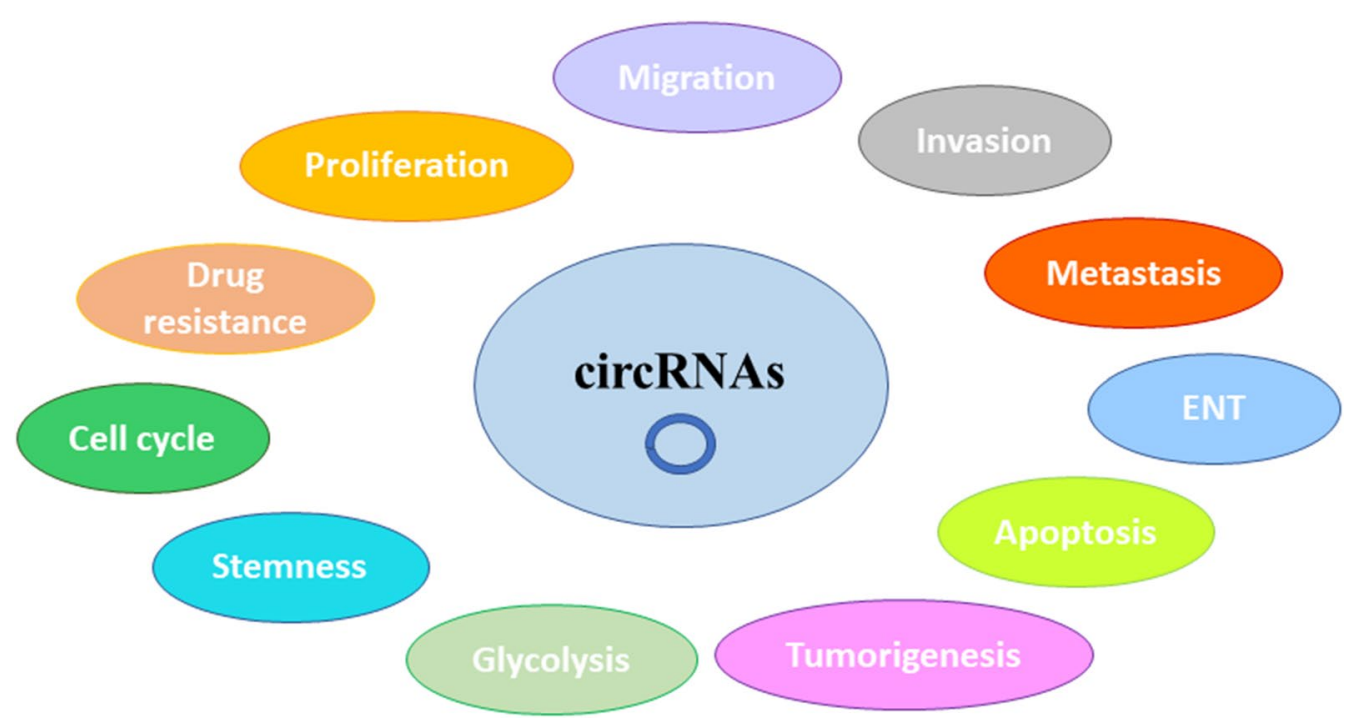

Fig. 1 The role of circRNAs in regulating cellular processes. CircRNAs play a critical role in the regulation of cell proliferation, cell migration and invasion, epithelial-mesenchymal transition (EMT), cell apoptotic death, tumorigenesis, glycolysis, stemness, cell cycle, and drug resistance in SMTs in children

miR-766. In vivo experiments also confirmed that overexpression of circ_0001105 could inhibit tumor growth. Similarly, Wu et al. [20] showed substantially increased expression of circTAD2A in OS tissues and cell lines. Occurrence and metastasis suggested that circTAD2AmiR-203a-3p/CREB3 could be used as a new target for the treatment of OS. CircMYO10 activated H4K16Ac in the promoter region of the $\beta$-protein/LEF1 target gene by regulating the miR370-3p/RUVBL1 axis, thereby activating the Wnt/ $\beta$-protein signal transduction pathway [21]. In OS cells, circRNA-0008717 could directly bind to miR203 and promote OS progression via sponge effect and upregulation of BMI-1 expression [22]. CircFAT1 promoted YAP1 expression in OS cells by sponging miR-375 [23]. Hsa_circ_0000282 acted as a competitive endogenous RNA and promoted OS cell proliferation by regulating miR-192/XIAP axis [24]. circ_0001649 can inhibit the viability and survival of U2OS and HOS cells by sponging miR-338-5p, miR-647 and miR-942, induce apoptosis and activate the STAT signaling pathway [25].

\section{Glioma}

Glioma is the most common primary central nervous system tumor, accounting for about half of all intracranial primary tumors. Most children get sick around 10 years of age. CircRNAs are known to promote angiogenesis in gliomas [26]. Studies have observed elevated levels of circ_002136 in gliomas, and its knockdown could suppress angiogenesis in gliomas. MiR-138-5p is known to target and regulate circ_002136 and reverse circ_002136-mediated angiogenesis of gliomas. Bioinformatic studies by $\mathrm{He}$ et al. identified the target gene SOX13 of miR-138-5p involved in angiogenesis. Functional analysis found that miR-138-5p inhibited SOX13mediated angiogenesis and SPON2 expression. SOX13 promoted FUS transcription to form a feedback loop that regulated glioma angiogenesis. FUS, as an RBP, promoted the generation of circRNA to regulate angiogenesis in gliomas. Also, studies by He et al. demonstrated the presence of a feedback loop in the angiogenesis of glioma, namely FUS (RBP), circ_002136 (CircRNA), miR-138-5p (MiRNA), SOX13 (transcription factor), and SPON2 Target genes, a multi-level molecular regulatory network. Other studies have shown that circRNA expression affects the cell cycle in glioma cells [27]. Targeted siRNAs-mediated knockdown of circHIPK3 has been shown to substantially suppress cell proliferation and invasion as well as arrested G0/G1 cell cycle while inducing apoptosis. After further experimentation, Jin et al. [28] speculated that circHIPK 3 affected cell proliferation, invasion, and metastasis of glioma cells through the circHIPK3/ miR-654/IGF2BP3 regulatory network. The experimental evidence suggested that circHIPK3 was a potential therapeutic target for the treatment of gliomas.

\section{Neuroblastoma}

Neuroblastoma (NB), a common extracranial tumor, is usually found in infants and young children. NB is a neuroendocrine tumor, which can originate from any nerve ridge of the sympathetic nervous system. Chen 
Table 1 Role of circRNAs in solid malignant tumors (SMTs) in children

\begin{tabular}{|c|c|c|c|c|c|}
\hline SMTs in children & CircRNA & Expression & Target gene/protein & Biological function & Ref \\
\hline \multirow[t]{7}{*}{ Osteosarcoma } & Circ_0001105 & Down & MiR-766/YTHDF2 & Inhibit proliferation, migration, and invasion & {$[1$} \\
\hline & CircTADA2A & Up & MiR-203a-3p/CREB3 & Promote progression and metastasis & {$[20$} \\
\hline & CircMYO10 & Up & MiR-370-3p/RUVBL1 & Promote proliferation and EMT & {$[21$} \\
\hline & Circ-0008717 & Up & MiR-203 & Promote proliferation, migration, and invasion & [22] \\
\hline & CircFAT1 & Up & MiR-375/YAP1 & Promote migration, invasion, and tumorigenesis & {$[23$} \\
\hline & Hsa_circ_0000282 & Up & miR-192/XIAP & Promote proliferation & {$[24$} \\
\hline & circ_0001649 & Down & MiR-338-5p/miR-647/miR-942 & Inhibit proliferation, migration; induce apoptosis & {$[2$} \\
\hline \multirow[t]{3}{*}{ Glioma } & Circ_002136 & Up & MiR-138-5p/SOX13 & Promote angiogenesis & {$[26$} \\
\hline & CircHIPK3 & Up & MiR-124-3p & $\begin{array}{l}\text { Promote proliferation, migration, and invasion; } \\
\text { inhibit apoptosis }\end{array}$ & {$[27$} \\
\hline & & & MiR-654/IGF2BP3 & Promote proliferation, migration, and invasion & {$[28$} \\
\hline \multirow[t]{3}{*}{ Neuroblastoma } & CircAGO2 & Up & & Promote proliferation, migration, and invasion & {$[2$} \\
\hline & CircCUX1 & Up & EWSR1/MAZ & Induce the aerobic glycolysis & {$[3$} \\
\hline & & Up & miR-16-5p/DMRT2 & $\begin{array}{l}\text { Promote proliferation, migration, invasion, and glyco- } \\
\text { lysis }\end{array}$ & {$[3$} \\
\hline \multirow[t]{3}{*}{ Hepatoblastoma } & Circ_0015756 & Up & MiR-1250-3p & Promote proliferation and invasion & {$[32$} \\
\hline & CircHMGCH1 & Up & MiR-503-5p/IGF2 & Promote proliferation & {$[3$} \\
\hline & CircSTAT3 & Up & MiR-29a/b/c-3p & Promote proliferation, invasion, migration, stemness & {$[3$} \\
\hline \multirow[t]{4}{*}{ Infantile hemangioma } & Hsa_circ_100933 & Up & & & {$[35$} \\
\hline & Hsa_circ_100709 & Up & & & {$[35$} \\
\hline & Hsa_circ_104310 & Down & & & {$[35$} \\
\hline & CircAP2A2 & Up & MiR-382-5p/VEGFA & Promote proliferation, migration, and invasion & {$[37$} \\
\hline Rhabdomyosarcoma & Circ-ZNF609 & Up & & Regulate G1-S progression & {$[38$} \\
\hline \multirow[t]{3}{*}{ Retinoblastoma } & Circ-0075804 & Up & E2F3 & Promote proliferation & {$[39$} \\
\hline & CircVAPA & Up & miR-615-3p/SMARCE1 & Promote proliferation, migration, and invasion & {$[40$} \\
\hline & Hsa_circ_0001649 & Up & AKT & $\begin{array}{l}\text { Promote proliferation, migration, and invasion; } \\
\text { inhibit apoptosis }\end{array}$ & {$[4$} \\
\hline Wilms'tumor & Circ_0017247 & Up & MiR-145 & Induce metastasis & {$[4$} \\
\hline \multirow[t]{2}{*}{ Medulloblastoma } & Circ-SKA3 & Up & & Promote the proliferation, migration and invasion & [43] \\
\hline & Circ-DTL & Up & & Promote the proliferation, migration and invasion & {$[4$} \\
\hline
\end{tabular}

et al. [29] found an upregulated expression of circAGO2 in NB tissues and cell lines. They speculated a relationship between circAGO2 and cancer growth, invasion, and metastasis and were able to experimentally prove the interaction between circAGO2 and HuR in cancer cells, followed by the activation of the latter. By promoting the HuR inhibitory function of the AGO2-miRNA complex, AGO2-miRNA-mediated gene silencing was suppressed, leading to increased target gene expression; thereby, promoting the occurrence and invasion of tumors. Thus, circAGO2 could help with the development of strategies to diagnose NB. They further found that circAGO2 knockout increased the survival probability of nude mice. This preliminary evidence suggested that circRNAs played an important role in the prognosis of NB. The experimental study by $\mathrm{Li}$ et al. [30] found that the introncontaining circular RNA (circ-CUX1) produced by the oncogene CUX1 was vital for the occurrence and invasion of NB. QRT-PCR analysis detected the upregulation of circCUX1 in NB tissues and cell lines. The upregulated circ-CUX1 bound to EWSR1 to promote its interaction with MYC-related zinc finger proteins to induce the aerobic glycolysis in NB. Subsequent functional studies found that circCUX1 had a carcinogenic role in the progression of NB. Transfected cell lines with stable circCUX1 expression exhibited enhanced glycolysis and reduced oxygen consumption. After stable transfection of circCUX1 in IMR32 cells, a substantial increase in growth, tumor weight, Ki-67 proliferation index, CD31positive microvessels, glucose uptake, lactate production, and ATP levels in nude mice was observed. Finally, they discovered that circ-CUX1 targeted miR-16-5p/DMRT2 signaling cascade to regulate the proliferation, migration, invasion, and glycolysis of NB cells [31]. 


\section{Hepatoblastoma}

Hepatoblastoma (HB), a malignant embryonic tumor, is a common liver tumor in children. Liu's study used gene sequencing to detect the differential expression of circRNA in the $\mathrm{HB}$ tissue and adjacent tissue [32]. They found a substantially upregulated expression of circ_0015756 in HB tissues. Functional studies found that circ_0015756 acted as a miR-1250-3p sponge to regulate HB cell function. Circ_0015756 knockdown reduced HB cell viability, proliferation, and invasion in vitro. They also found considerably elevated circ_0015756 expression in the peripheral blood of patients with HB. Similarly, Zhen et al. [33] also found a substantially heightened circHMGCS1 expression in HB tissues of 64 hepatectomy patients and normal liver tissues, as well as observed that circHMGCS1 was regulated by the "sponge" miR503-5p, the expression of IGF2 and IGF1R, and affected the downstream PI3K-Akt signaling pathway, regulated $\mathrm{HB}$ cell proliferation. This suggested that circHMGCS1 could be a promising therapeutic target and prognostic marker for HB. Liu et al. [34] found that circ-STAT3 acted as an miR-29a/b/c-3p sponge to elevate STAT3 and Gli2 expression with Gli2 as the transcription factor for circ-STAT3. Circ-STAT3 facilitated cell proliferation, invasion, migration, stemness, and tumor growth in $\mathrm{HB}$ by upregulating STAT3 and Gli2.

\section{Infantile hemangioma}

Infantile hemangioma $(\mathrm{IH})$ is a congenital benign tumor or vascular malformation induced by the proliferation of hemangioblasts during embryo formation and is common in skin and soft tissues. It is commonly detected either at the time of birth or shortly after birth. Fu et al. [35] were the first ones to analyze circRNAs in IH, identified the circRNAs that were differentially expressed, and identified 234 upregulated, and 374 downregulated circRNAs. Among them, hsa_circ_100933 and hsa_ circ_100709 were upregulated, and hsa_circ_104310 was downregulated. Similarly, Li et al. [36] conducted a study on the proliferating capillary hemangioma and matching normal skin tissues, obtained from 3 children, and found 249 differentially expressed circRNAs (DE-circRNAs) via qRT-PCR. There were 124 upregulated and 125 downregulated circRNAs in the $\mathrm{IH}$ tissue. The expressions of hsa_cicRNA_001885 and hsa_cicRNA_006612 in hemangioma tissue are 12.33 times and 7.13 times higher than that of normal skin, respectively. They also analyzed the source genes of DE-circRNAs via GO and KEGG pathway analysis. GO analysis showed that genes derived from DE-circRNAs are mainly involved in the generation of cellular components, protein binding, and chemical components in cells. KEGG pathway analysis showed that the genes of origin of DE-circRNAs were mainly related to information exchange between cells. CircAP2A2 was highly expressed in $\mathrm{IH}$ [35]. Downregulated expression of circAP2A2 could substantially inhibit growth, proliferation, invasion, and migration of IH cells. Yuan et al. [37] performed bioinformatics analysis and functional studies and showed that circAP2A2 regulated VEGFA expression through sponge miR-382-5p, promoting the development of hemangioma. Therefore, circAP2A2/miR-382-5p/ VEGFA axis might provide new ideas for the treatment of IH.

\section{Rhabdomyosarcoma}

Rhabdomyosarcoma (RMS) is the most common type of soft tissue sarcoma in children. It is a malignant tumor that originates from striated muscle cells or mesenchymal cells that differentiate into striated muscle cells. Rossi et al. [38] studied the expression and function of circ-ZNF609 in children with RMS to understand the role of circ-ZNF609 in cell cycle regulation. They found that circ-ZNF609 was mainly upregulated in embryonic and alveolar RMS biopsies. Also, circ-ZNF609 knockout in embryonic RMS-derived cell lines resulted in a substantial decrease in p-Akt protein levels, vital for the cell cycle. Thus, it was hypothesized that circ-ZNF609 acted as a new regulator of cell proliferation pathways by resisting p-Akt proteasome-dependent degradation. Also, Rossi et al. found that circ-ZNF609 downregulated cell cycle-related genes and upregulated innate immune genes via RNA sequence analysis of human primary myoblasts lacking circ-ZNF609, indicating a close relationship between circ-ZNF609 and RMS cell proliferation.

\section{Retinoblastoma}

Retinoblastoma (RB), a malignant tumor derived from photoreceptor precursor cells, is usually found in children under 3 years old, with family genetic predisposition. Circ-0075804 promotes RB cell proliferation by binding to heterogeneous ribonucleoprotein K (HNRNPK), thereby increasing the stability of E2F3 mRNA, which indicates that circ-0075804 may become a therapeutic target for RB patients [39]. Xu found that circVAPA was upregulated in human RB specimens and $\mathrm{RB}$ cell lines through qRT-PCR, and was associated with the poor prognosis of RB patients. Knockout of circVAPA can inhibit the malignant phenotype of RB. After studying the mechanism, he found that miR-615-3p can reverse the RB cell effect induced by circVAPA, and circVAPA positively regulates the downstream oncogene SMARCE1 through miR-615-3p. In addition, in vivo nude mice tumor formation experiments confirmed this finding [40]. Xing et al. found that the down-regulation 
of the circular RNA hsa_circ_0001649 indicates that the prognosis of retinoblastoma is poor, and it regulates cell proliferation and apoptosis through the AKT/mTOR signaling pathway [41]. Which indicates that strengthening the effect of hsa_circ_0001649 may be a potential treatment strategy for RB.

\section{Other SMTs in children}

Children's SMTsare not only limited to the above-mentioned types, but also Wilms' tumor, medulloblastoma, ependymoma, etc. Studies have found that circ_0017247 induces Wilms' tumor metastasis through targeting miR145 [42]. circ-SKA3 and circ-DTL promote the proliferation, migration and invasion of medulloblastoma cells by regulating the expression of host genes. This shows that circ-SKA3 and circ-DTL have key carcinogenic effects in the occurrence and development of medulloblastoma [43]. Ahmadov et al. found that ependymoma specifically upregulated circRNA derived from RMST, LRBA, WDR78, DRC1 and BBS9 genes through Next Generation Sequencing, but their results still need experiments to prove [44].

\section{Conclusion}

With the advancements in high-throughput sequencing techniques, extensive studies are being conducted to understand the role of circRNAs in various diseases. Due to the specific structure of circRNA, it is highly conserved and abundantly present in cells. Currently, it can be regarded as a research hotspot. Particularly, the relationship between circRNA and tumors cannot be ignored. Several studies have shown that circRNAs play a vital role in tumor occurrence, proliferation, metastasis, invasion, EMT, apoptosis, and cell cycle. The dysregulated circRNAs between SMTs in children and normal cells/tissues may participate in regulating the inhibition and promotion of these SMTs in children, suggesting that circRNAs could serve as therapeutic targets and biomarkers for clinical diagnosis. However, the above assumptions continue to present substantial difficulties and enormous challenges in clinical practice, and more studies on the significance, efficiency, security, and reliability of these approaches are needed. Thus, we expect that this review will increase the comprehension of the principal functions of many circRNAs and their multiple regulatory hubs in the progression of SMTs in children. We are optimistic that the use of sequencing technologies will elucidate roles of circRNAs implicated in SMTs in children tumorigenesis may eventually accelerate the clinical application of circRNAs for use in diagnosis, treatment, and prognosis evaluation.

\section{Authors' contributions}

Data collection and organization-YJB, YL; write the paper-YJB; conceptualization, supervision, manuscript reviewing and editing-LHT. All authors have read and approved the final manuscript.

\section{Funding}

This work was funded by the Shandong Provincial Natural Science Foundation (ZR2020MH213)

\section{Availability of data and materials \\ Not applicable.}

\section{Declarations}

Ethics approval and consent to participate

Not applicable.

\section{Consent for publication}

Not applicable.

\section{Competing interests}

Not applicable.

\section{Author details}

${ }^{1}$ Qingdao University, Qingdao, Shandong, China. ${ }^{2}$ Surgical Center of Women and Children's Hospital, Qingdao University, No. 6, Tongfu Rd, Shibei District, Qingdao 266011, Shandong, China.

Received: 26 March 2021 Accepted: 27 May 2021

Published online: 11 June 2021

\section{References}

1. Bao PP, Zheng $Y$, Jin F. Research progress on environmental risk factors of malignant tumors in children. Environ Occupa Med. 2008;25(2):190-4.

2. Rebecca L, Siegel Kimberly D, Miller Ahmedin J. Cancer Statistics. CA Cancer J Clin. 2019;69:7-34.

3. Jeck WR, Sharpless NE. Detecting and characterizing circular RNAs. Nat Biotechnol. 2014;32(5):453-61.

4. Sanger HL, Klotz G, Riesner D, et al. Viroids are single-stranded covalently closed circular RNA molecules existing as highly base-paired rod-like structures. Proc Natl Acad Sci USA. 1976;73(11):3852-6.

5. Salzman J, Gawad C, Wang PL, et al. Circular RNAs are the predominant transcript isoform from hundreds of human genes in diverse cell types. PLoS One. 2012;7(2):e30733.

6. JeckWR, Sorrentino JA, Wang K, et al. Circular RNAs are abundant, conserved, and associated with ALU repeats. RNA. 2013;19(2):141-57.

7. Chen B, Huang S. Circular RNA: an emerging non-coding RNA as a regulator and biomarker in cancer. Cancer Lett. 2018. https://doi.org/10.1016/j. canlet.2018.01.011.

8. Huang C, Shan G. What happens at or after the transcription- Insights into circRNA biogenesis and function. Transcription. 2015. https://doi.org/ 10.1080/21541264.2015.1071301.

9. Li Z, Huang C, Bao C, et al. Exon-intron circular RNAs regulate transcription in the nucleus. Nat Struct Mol Biol. 2015;22(3):256-64.

10 Lu Z, Filonov GS, Noto JJ, et al. Metazoan tRNA introns generate stable circular RNAs in vivo. RNA. 2015. https://doi.org/10.1261/rna.052944.115.

11. Memczak S, Jens M, Elefsinioti A, et al. Circular RNAs are a large class of animal RNAs with regulatory potency. Nature. 2013;495(7441):333-8.

12. Zheng $Q$, Bao $C$, Guo W, et al. Circular RNA profiling reveals an abundant circHIPK3 that regulates cell growth by sponging multiple miRNAs. Nat Commun. 2016;7:11215.

13. Chao CW, Chan DC, Kuo A, et al. The mouse formin (Fmn) gene: abundant circular RNA transcripts and gene-targeted deletion analysis. Mol Med. 1998:4(9):614.

14. Chen Y, Yang F, Fang E, et al. Circular RNA circAGO2 drives cancer progression through facilitating HuR-repressed functions of AGO2-miRNA complexes. Cell Death Differ. 2019;26(7):1346. 
15. Ashwal-Fluss R, Meyer M, Pamudurti N, et al. CircRNA biogenesis competes with Pre-mRNA splicing. Mol Cell. 2014;56(1):55-66.

16. Kos A, Dijkema R, Arnberg AC, et al. The hepatitis delta $(\delta)$ virus possesses a circular RNA. Nature. 1986;323(6088):558-60

17 Guo JU, Agarwal V, Guo H, Bartel DP. Expanded indentification and characterization of mammalian circular RNAs. Genome Biol. 2014;15(7):409.

18. Legnini I, Di Timoteo G, Rossi F, et al. Circ-ZNF609 is a circular RNA that can be translated and functions in myogenesis. Mol Cell. 2017;66(1):2237.e9.

19. Yang J, Han Q, Li C, et al. Circular RNA circ_0001105 inhibits progression and metastasis of osteosarcoma by sponging miR-766 and activating YTHDF2 expression. OncoTargets Ther. 2020;13:1723.

20. Wu Y, Xie Z, Chen J, et al. Circular RNA circTADA2A promotes osteosarcoma progression and metastasis by sponging miR-203a-3p and regulating CREB3 expression. Mol Cancer. 2019;18(1):73.

21. Chen J, Liu G, Wu Y, et al. CircMYO10 promotes osteosarcoma progression by regulating miR-370-3p/RUVBL1 axis to enhance the transcriptional activity of $\beta$-catenin/LEF1 complex via effects on chromatin remodeling. Mol Cancer. 2019;18(1):1-24.

22. Zhou X, Natino D, Qin Z, et al. Identification and functional characterization of circRNA-0008717 as an oncogene in osteosarcoma through sponging miR-203. Oncotarget. 2018;9(32):22288.

23. Liu G, Huang K, Jie Z, et al. CircFAT1 sponges miR-375 to promote the expression of Yes-associated protein 1 in osteosarcoma cells. Mol Cancer. 2018;17(1):170.

24. Li H, He L, Tuo Y, et al. Circular RNA hsa_circ_0000282 contributes to osteosarcoma cell proliferation by regulating miR-192/XIAP axis. BMC Cancer. 2020;20(1):1-10.

25. Sun D, Zhu D. Circular RNA hsa_circ_0001649 suppresses the growth of osteosarcoma cells via sponging multiple miRNAs. Cell Cycle. 2020;19(20):2631-43.

26. He Z, Ruan X, Liu X, et al. FUS/Circ_002136/miR-138-5p/SOX13 feedback loop regulates angiogenesis in Glioma. J Exp Clin Cancer Res. 2019;38(1):65.

27. Hu D, Zhang Y. Circular RNA HIPK3 promotes glioma progression by binding to miR-124-3p. Gene. 2019;690:81-9.

28. Jin P, Huang Y, Zhu P, et al. CircRNA circHIPK3 serves as a prognostic marker to promote glioma progression by regulating miR-654/IGF2BP3 signaling. Biochem Biophys Res Commun. 2018;503(3):1570-4.

29. Chen $Y$, Yang F, Fang E, et al. Circular RNA circAGO2 drives cancer progression through facilitating HuR-repressed functions of AGO2-miRNA complexes. Cell Death Differ. 2019;26(7):1346.

$30 \mathrm{Li} \mathrm{H}$, Yang F, Hu A, et al. Therapeutic targeting of circ-CUX1/EWSR1/MAZ axis inhibits glycolysis and neuroblastoma progression. EMBO Mol Med. 2019. https://doi.org/10.15252/emmm.201910835.

31 Zhang X, Zhang J, Liu Q, et al. Circ-CUX1 accelerates the progression of neuroblastoma via miR-16-5p/DMRT2 Axis. Neurochem Res. 2020. https://doi.org/10.1007/s11064-020-03132-w.
32 Liu BH, Zhang BB, Liu XQ, et al. Expression profiling identifies circular RNA signature in hepatoblastoma. Cell Physiol Biochem. 2018. https://doi.org/ 10.1159/000487163.

33. Zhen N, Gu S, Ma J, et al. CircHMGCS1 promotes hepatoblastoma cell proliferation by regulating the IGF signaling pathway and glutaminolysis. Theranostics. 2019;9(3):900-19.

34. Liu Y, Song J, Liu Y, et al. Transcription activation of circ-STAT3 induced by Gli2 promotes the progression of hepatoblastoma via acting as a sponge for miR-29a/b/c-3p to upregulate STAT3/Gli2. J Exp Clin Cancer Res. 2020;39(1):1-14.

35. Cong F, Renrong L, Guangqi X, et al. Circular RNA profile of infantile hemangioma by microarray analysis. PLOS One. 2017;12(11):e0187581.

36 Jun L, Qian L, Ling C, et al. Expression profile of circular RNAs in infantile hemangioma detected by RNA-Seq. Medicine. 2018;97(21):e10882.

37. Yuan $X, X u Y$, Wei $Z$, et al. CircAP2A2 acts as a ceRNA to participate in infantile hemangiomas progression by sponging miR-382-5p via regulating the expression of VEGFA. J Clin Lab Anal. 2020. https://doi.org/10. 1002/jcla.23258.

38. Rossi F, Legnini I, Megiorni F, et al. Circ-ZNF609 regulates G1-S progression in rhabdomyosarcoma. Oncogene. 2019;38(20):3843-54.

39. Zhao W, Wang S, Qin T, et al. Circular RNA (circ-0075804) promotes the proliferation of retinoblastoma via combining heterogeneous nuclear ribonucleoprotein $\mathrm{K}$ (HNRNPK) to improve the stability of E2F transcription factor 3 E2F3. J Cell Biochem. 2020. https://doi.org/10.1002/jcb. 29631.

40. Xu Q. The Potential tumor promotional role of circVAPA in retinoblastoma via regulating miR-615-3p and SMARCE1. Onco Targets Ther. 2020;13:7839.

41. Xing $L$, Zhang $L$, Feng $Y$, et al. Downregulation of circular RNA hsa_ circ_0001649 indicates poor prognosis for retinoblastoma and regulates cell proliferation and apoptosis via AKT/mTOR signaling pathway. Biomed Pharmacother. 2018;105:326-33.

42. Du A. Circ_0017247 induces Wilms' tumor metastasis through targeting miR-145. Minerva Medica. 2020.https://doi.org/10.23736/S0026-4806.20. 06845-7.

43. LvT, Miao YF, Jin K, et al. Dysregulated circular RNA s in medulloblastoma regulate proliferation and growth of tumor cells via host genes. Cancer Med. 2018:7(12):6147-57.

44 Ahmadov U, Bendikas MM, Ebbesen KK, et al. Distinct circular RNA expression profiles in pediatric ependymomas. Brain Pathol. 2020. https://doi. org/10.1111/bpa.12922.

\section{Publisher's Note}

Springer Nature remains neutral with regard to jurisdictional claims in published maps and institutional affiliations.
Ready to submit your research? Choose BMC and benefit from:

- fast, convenient online submission

- thorough peer review by experienced researchers in your field

- rapid publication on acceptance

- support for research data, including large and complex data types

- gold Open Access which fosters wider collaboration and increased citations

- maximum visibility for your research: over 100M website views per year

At BMC, research is always in progress.

Learn more biomedcentral.com/submissions 